\title{
Review of: "The effectiveness of diaphragmatic breathing relaxation training for improving sleep quality among nursing staff during the COVID-19 outbreak: a before and after study"
}

Yicheng Long ${ }^{1}$

1 Central South University

Potential competing interests: The author(s) declared that no potential competing interests exist.

This is an interesting and valuable paper, well done! But there is a minor point. In the abstract it was mentioned "First-line nurses achieved significant reductions in global sleep quality $(p<0.01)$ ", which might be misunderstanding. After intervention, the nurses' PSQI scores were reduced, but it means an improved sleep quality. 